\title{
SIMPLE AND RAPID LIQUID CHROMATOGRAPHY METHOD FOR DETERMINATION OF RIFABUTIN IN PLASMA
}

\author{
Hemanth Kumar AK, Sudha V, Ramachandran G \\ National Institute for Research in Tuberculosis, Chetput, Chennai, India
}

\begin{abstract}
A high performance liquid chromatographic method for determination of rifabutin in human plasma was developed. The method involved deproteinisation of the sample with acetonitrile and analysis of the supernatant using a reversed-phase $C_{18}$ column $(250 \mathrm{~mm})$ and UV detection at a wavelength of $265 \mathrm{~nm}$. The assay was specific for rifabutin and linear from 0.025 to $10.0 \mu \mathrm{g} / \mathrm{ml}$. The relative standard deviation of intra- and inter-day assays was lower than $10 \%$. The method was able to remove interfering materials in plasma, yielding an average recovery of rifabutin from plasma of $101 \%$. Due to its simplicity, the assay can be used for pharmacokinetic studies of rifabutin.
\end{abstract}

\section{INTRODUCTION}

Patients' adherence to anti TB treatment may be he rifamycin class of compounds, rifampicin, rifapentine and rifabutin play a key role in the treatment of tuberculosis. The rifamycins are known to be potent inducers of the hepatic CYP450 enzyme system, but vary in their potential as CYP450 inducers, with rifampicin being most potent, rifapentine intermediate, and rifabutin being much less active. Rifabutin has been shown to be as effective against tuberculosis as rifampici ${ }^{1,2}$ and is the preferred rifamycin in situations were rifampicin is contraindicated, such as during concomitant use of protease inhibitors. ${ }^{3}$

A few methods have been described for estimation of plasma rifabutin. The method of Lewis et al involves a cumbersome sample preparation procedure, using one $\mathrm{ml} \mathrm{plasma}^{4}$, while other methods require special columns $s^{5,6}$ and column switching. ${ }^{7}$ We developed and validated a simple

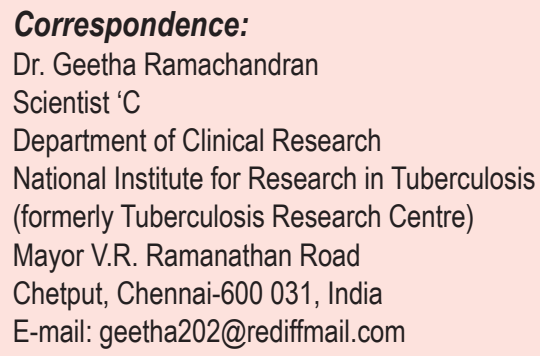

and rapid assay procedure for estimation of rifabutin in plasma using rifampicin as an internal standard.

\section{METHODOLOGY}

Pure rifabutin and rifampicin powders from Sigma Chemical Company, MO, USA, Acetonitrile (HPLC grade) from Merck (India) and potassium dihydrogen orthophosphate from Qualigens (India) were used. Deionized water was processed through a Milli-Q water purification system (Millipore, USA). Pooled human plasma was obtained from Lions Blood Bank, Chennai, India.

The HPLC system (Shimadzu Corporation, Kyoto, Japan) consisted of two pumps (LC-10ATvp), diode array detector (SPD-M10Avp) and auto-sampler with built-in system controller (SIL-HTA). Class VP-LC workstation was used for data collection and acquisition. The analytical column was a $C_{18}, 250$ x $4.6 \mathrm{~mm}$ ID, $5 \mu$ particle size (Lichrospher $100 \mathrm{RP}$ $18 \mathrm{e}$, Merck, Germany) protected by a compatible guard column.

The mobile phase consisted of $50 \mathrm{mM}$ phosphate buffer, pH 4.2 (adjusted with $1 \mathrm{~N} \mathrm{HCl}$ ) and acetonitrile $(53: 47 \mathrm{v} / \mathrm{v})$. Prior to preparation of the mobile phase, the phosphate buffer and acetonitrile were degassed separately using a Millipore vacuum pump. The UV detector was set 
at a wavelength of $265 \mathrm{~nm}$. The chromatogram was run for 10 minutes at a flow rate of $1.2 \mathrm{ml} / \mathrm{min}$ at ambient temperature. Unknown concentrations were derived from linear regression analysis of the peak height ratios (analyte/internal standard) vs. concentration curve. The linearity was verified using estimates of correlation coefficient ( $r$ ).

A stock standard $(1 \mathrm{mg} / \mathrm{ml})$ was prepared by dissolving rifabutin in methanol. The working standards of rifabutin in concentrations ranging from 0.025 to $10.0 \mu \mathrm{g} / \mathrm{ml}$ were prepared in human plasma.

To $200 \mu$ each of calibration standards and test samples, $20 \mu \mathrm{l}$ of rifampicin (internal standard) was added at a concentration of $20 \mu \mathrm{g} / \mathrm{ml}$. This was mixed with $300 \mu \mathrm{l}$ of acetonitrile, the contents were vortexed vigorously, and centrifuged at 10,000 rpm for 10 minutes. Three hundred microlitres of the clear supernatant was taken into the test tube, evaporated to dryness in a nitrogen evaporator (turbo vapor) for 20 minutes. The dried residue was reconstituted in $200 \mu$ l of mobile phase; $100 \mu$ was injected into the HPLC column

The accuracy and linearity of rifabutin standards were evaluated by analysing a set of standards ranging from 0.025 to $10.0 \mu \mathrm{g} / \mathrm{ml}$. The within day and between day variations were determined by processing each standard concentration in duplicate for six consecutive days.

In order to evaluate the precision of the method, three different concentrations of rifabutin (1.3, 5.5 and $10.0 \mu \mathrm{g} / \mathrm{ml}$ ) were prepared in pooled human lasma and analysed in duplicate on three consecutive days.

Varying concentrations of rifabutin $(0.3,4.5$ and $9.0 \mu \mathrm{g} / \mathrm{ml}$ ) were prepared in drug-free human plasma and extracted as described above along with the internal standard. The percentage of the drug recovered from the plasma samples was determined by comparing the peak height ratio after extraction with those of unextracted methanolic solutions containing same concentrations of rifabutin as in plasma. Recovery experiments were carried out on three different occasions.
Interference from endogenous compounds was investigated by analysing blank plasma samples obtained from six each of male and female subjects. Interference from certain anti-tuberculosis drugs such as, isoniazid, pyrazinamide, rifapentine, ethambutol, streptomycin and certain antiretroviral drugs, namely, nevirapine, efavirenz, zidovudine, tenofovir, didanosine, stavudine, lamivudine, lopinavir, ritonavir, indinavir and saquinavir at a concentration of $10 \mu \mathrm{g} / \mathrm{ml}$ was also evaluated.

Serial blood samples at 2, 4 and 6 hours post dosing were drawn in heparinized containers from a HIV-infected child with tuberculosis (age - 12 years; sex - male; body weight - 22kg) who was receiving rifabutin (150mg thrice weekly) as part of the treatment regimen. All the blood samples were centrifuged and plasma was separated and stored at $-20^{\circ} \mathrm{C}$ until assay. Estimation of plasma rifabutin was undertaken within 24 to 48 hours of blood collection. Informed written consent was obtained from the parent before blood draws were made.

\section{RESULTS}

Under the chromatographic conditions described above, rifabutin was well separated as seen in the representative chromatograms (Figure 1a,b). The retention times of the internal standard and rifabutin were 2.5 and 8.6 minutes respectively. Blank plasma samples did not give any peak at the retention times of rifabutin (Figure 1c). Chromatogram of a patient's sample containing rifabutin and extracted following the same procedure as standards is shown in Figure $1 \mathrm{~d}$. The lowest concentration of rifabutin gave a discrete peak at 8.7 minutes (Figure 1a).

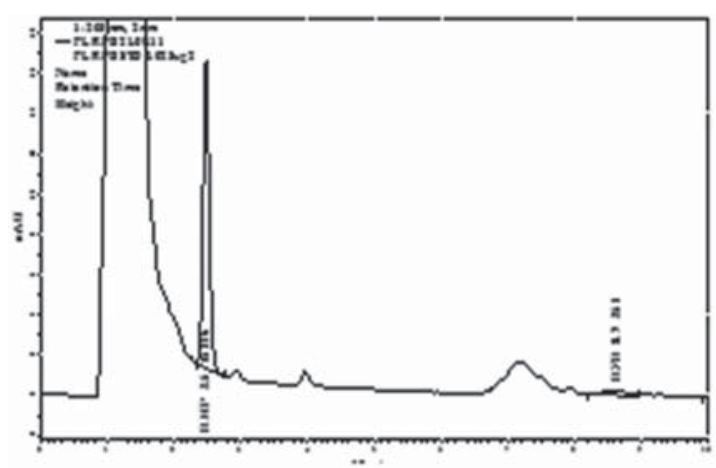

Figure 1a: Chromatogram of extracted rifabutin plasma standard $0.025 \mu \mathrm{g} / \mathrm{ml}$ (contains internal standard - 20 $\mu$ of rifampicin added at a concentration of $20 \mu \mathrm{g} / \mathrm{ml}$ ) 


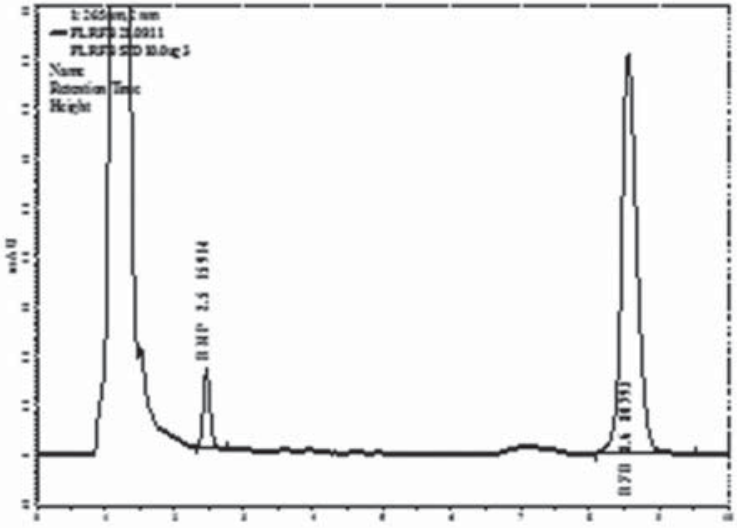

Figure 1b: Chromatogram of extracted rifabutin plasma standard $10.0 \mu \mathrm{g} / \mathrm{ml}$ (contains internal standard - $20 \mu$ of rifampicin added at a concentration of $20 \mu \mathrm{g} / \mathrm{ml}$ )

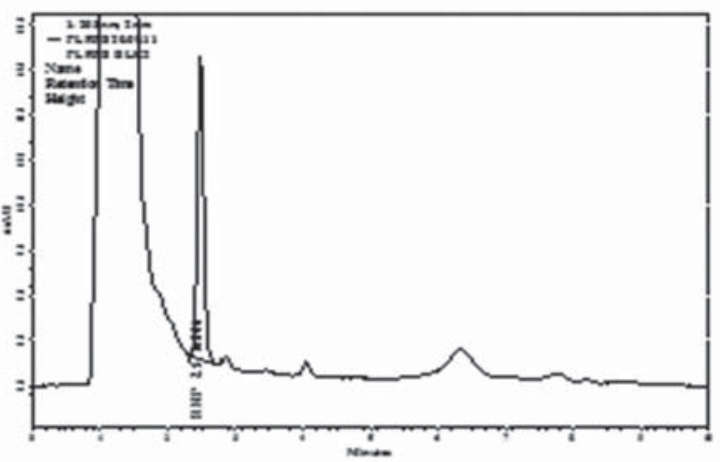

Figure 1c: Chromatogram of extracted blank plasma (contains internal standard - $20 \mu$ of rifampicin added at a concentration of $20 \mu \mathrm{g} / \mathrm{ml}$ )

No endogenous substances or first-line antituberculosis drugs such as isoniazid, pyrazinamide, ethambutol, streptomycin or antiretroviral drugs such as nevirapine, efavirenz, zidovudine, tenofovir, didanosine, stavudine, lamivudine, lopinavir, ritonavir, indinavir and saquinavir interfered with the rifabutin chromatogram.

Rifabutin concentrations ranging from 0.025-10.0 $\mu \mathrm{g} / \mathrm{ml}$ were checked for linearity. The calibration curve parameters of rifabutin from six individual experiments for standard concentrations ranging from 0.025 to $10.0 \mu \mathrm{g} / \mathrm{ml}$ showed a linear relationship between peak height ratio and concentrations. The mean $( \pm S D)$ correlation coefficient, slope and intercept values were $0.9993 \pm 0.0008,0.6685$ \pm 0.2758 and $-0.0225 \pm 0.016$ respectively. The linearity and reproducibility of the various standards used for constructing calibration graphs for plasma rifabutin are given in Table 1. The within-day and between-day percent variation (CV) for standards

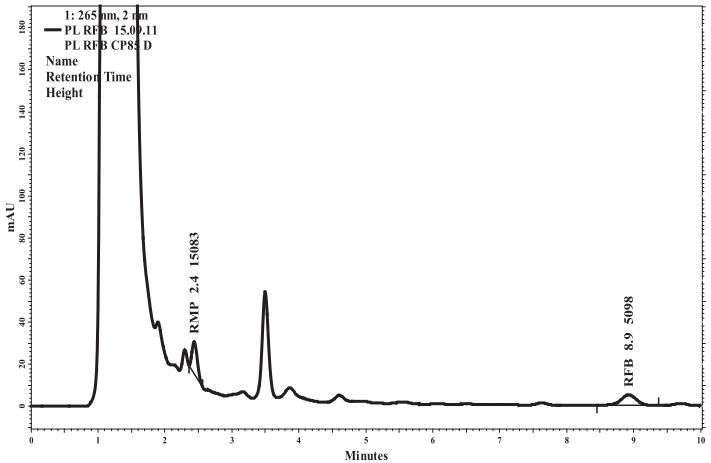

Figure 1d: Chromatogram of extracted rifabutin from a patient's plasma sample (contains internal standard - 20 $\mu \mathrm{l}$ of rifampicin added at a concentration of $20 \mu \mathrm{g} / \mathrm{ml}$ )

containing 0.025 to $10.0 \mu \mathrm{g} / \mathrm{ml}$ ranged from 1.0 to $9.1 \%$ and 2.1 to $9.1 \%$ respectively.

The reproducibility of the method was further evaluated by analysing three plasma samples containing different concentrations of rifabutin. The RSD for these samples ranged from 2.7 to $5.3 \%$ (Table 2). The \% variations from the actual concentrations ranged from 98 to $108 \%$. The Limit of Quantification (LOQ) was $0.025 \mu \mathrm{g} / \mathrm{ml}$. The percent recovery of rifabutin from plasma ranged from 98 to $100 \%$. The concentrations of rifabutin in serial blood samples collected at 2, 4 and 6 hours post dosing were $0.86,0.82$ and $0.17 \mu \mathrm{g} / \mathrm{ml}$; the peak concentration being $0.86 \mu \mathrm{g} / \mathrm{ml}$.

\begin{tabular}{|c|c|c|}
\hline \multirow{2}{*}{$\begin{array}{c}\text { Standard } \\
\text { Concentration } \\
(\mu \mathrm{g} / \mathrm{ml})\end{array}$} & \multicolumn{2}{|c|}{ Mean peak height ratio \pm SD $(\% \mathrm{CV})$} \\
\hline & Within day $(n=6)$ & Between day $(n=6)$ \\
\hline 0.025 & $0.011 \pm 0.001(9.1)$ & $0.01 \pm 0.001(8.9)$ \\
\hline 0.05 & $0.025 \pm 0.002(7.1)$ & $0.021 \pm 0.002(9.1)$ \\
\hline 0.1 & $0.047 \pm 0.002(4.5)$ & $0.043 \pm 0.003(7.8)$ \\
\hline 0.5 & $0.240 \pm 0.002(1.0)$ & $0.231 \pm 0.010(4.5)$ \\
\hline 1.0 & $0.488 \pm 0.019(3.9)$ & $0.470 \pm 0.022(4.8)$ \\
\hline 2.5 & $1.174 \pm 0.052(4.4)$ & $1.163 \pm 0.049(4.3)$ \\
\hline 5.0 & $2.438 \pm 0.090(3.7)$ & $2.377 \pm 0.147(6.2)$ \\
\hline 10.0 & $4.926 \pm 0.163(3.3)$ & $4.926 \pm 0.103(2.1)$ \\
\hline
\end{tabular}

\begin{tabular}{|c|c|}
\hline \multicolumn{2}{|c|}{ Table 2. Precision of plasma rifabutin assay } \\
\hline $\begin{array}{c}\text { Actual Conc. } \\
(\mu \mathrm{g} / \mathrm{ml})\end{array}$ & $\begin{array}{c}\text { Found Conc. } \\
(\mu \mathrm{g} / \mathrm{ml}) \\
\text { Mean } \pm \text { SD }(\% \mathrm{RSD})\end{array}$ \\
\hline 1.3 & $1.27 \pm 0.06(4.6)$ \\
\hline 5.5 & $5.38 \pm 0.29(5.3)$ \\
\hline 10 & $9.98 \pm 0.27(2.7)$ \\
\hline
\end{tabular}




\section{DISCUSSION}

A few HPLC methods have been described to measure rifabutin concentrations in plasma for pharmacokinetic studies. The method described by Lewis et al requires one $\mathrm{ml}$ plasma, and a lengthy sample pretreatment procedure ${ }^{4}$, while the methods of Lau et al and Gatti et al have employed special columns, which could be relatively expensive. ${ }^{5,6}$ The method of Bartels et al requires column switching, which could be cumbersome. ${ }^{7}$ The present method has the advantages of being rapid (run time is only 10 minutes), employing a simple one-step sample preparation step and using a small sample volume (300 microlitres), without any loss of analyte. The use of rifampicin as internal standard helped in monitoring the recovery of rifabutin from plasma.

The method was quite robust as evidenced by a discrete peak denoting rifabutin. The percent variations (within-day and between-day) for the standards were below $10 \%$. The method reliably eliminated interfering material from plasma, yielding an acceptable range of recovery. Further, data from the accuracy and precision experiments yielded satisfactory results.

In view of its potent antimycobacterial activity, rifabutin is likely to be used in the treatment of tuberculosis along with anti-tuberculosis and antiretroviral drugs. It therefore becomes necessary to rule out interference of both these classes of drugs in the assay of rifabutin and establish the specificity of the method. The method was highly specific for rifabutin; drugs such as isoniazid, pyrazinamide, ethambutol, streptomycin or antiretroviral drugs such as nevirapine, efavirenz, zidovudine, tenofovir, didanosine, stavudine, lamivudine, lopinavir, ritonavir, indinavir and saquinavir or any endogenous substance interfered in the assay.

When this method was applied to estimate rifabutin in plasma samples collected from a child, we obtained a peak concentration of $0.86 \mu \mathrm{g} / \mathrm{ml}$, which was within the therapeutic range of rifabutin. ${ }^{8}$ Thus the method spans the range of clinical interest and could be applied to pharmacokinetic studies in both adults and children.
In conclusion, a sensitive, specific and validated method for quantitative determination of rifabutin in plasma is described. This simple, rapid, accurate and reproducible method utilises a single step extraction. The chromatogram yields a wellresolved peak for rifabutin with good intra- and inter-day precision. The easy sample preparation and small sample size makes this assay highly suitable for pharmacokinetic studies of rifabutin in tuberculosis patients.

\section{REFERENCES}

1. Schwander S, Rusch-Gerdes S, Mateega A, Lutalo T, Tugume S, Kityo $C$, et al. A pilot study of antituberculosis combinations comparing rifabutin with rifampicin in the treatment of HIV-1 associated tuberculosis. A single-blind randomized evaluation in Ugandan patients with HIV-1 infection and pulmonary tuberculosis. Tubercle Lung Dis. 1995;76:210-18.

2. Gonzalez-Montaner LJ, Natal S, Yonchaiyud P, Olliaro P. Rifabutin for the treatment of newlydiagnosed pulmonary tuberculosis: a multinational, randomized, comparative study versus Rifampicin. Rifabutin Study Group. Tubercle Lung Dis. 1994;75:341-47.

3. Burman WJ, Gallicano K, Peloquin CA. Therapeutic implications of drug interactions in the treatment of HIV-related tuberculosis. Clin Infect Dis. 1999;28:419-30.

4. Lewis RC, Hatfield NZ, Narang PK. A sensitive method for quantitation of rifabutin and its desacetyl metabolite in human biological fluids by HPLC. Pharm Res. 1991;8:1434-40.

5. Lau YY, Hanson GD, Carel BJ. Determination of rifabutin in human plasma by high performance liquid chromatography with ultraviolet detection. J Chromatogr B Biomed Appl. 1996;676:125-30.

6. Gatti G, De Pascalis CR, Miletich F, Casazza $R$, Bassetti D. Specific high performance liquid chromatography assay for determination of rifabutin plasma concentration following Extrelut column extraction. J Chromatogr B Biomed Sci Appl. 1999;728:233-39.

7. Bartels $H$, Bartels R. Determination of rifabutin by high performance liquid chromatography using on-line concentration and column switching. J Chromatogr B Biomed Appl. 1996;686:235-40.

8. Peloquin CA. Therapeutic drug monitoring in the treatment of tuberculosis. Drugs 2002;62:2169-83. 